\title{
Correction to: On Nonlocal Variational and Quasi-Variational Inequalities with Fractional Gradient
}

\author{
José Francisco Rodrigues ${ }^{1} \cdot$ Lisa Santos ${ }^{2}$
}

Published online: 25 March 2021

(c) Springer Science+Business Media, LLC, part of Springer Nature 2021

\section{Correction to: Appl Math Optim https://doi.org/10.1007/s00245-019-09610-0}

${ }^{1}$ We correct the norm of the usual fractional Sobolev space

$$
H_{0}^{\sigma}(\Omega), \quad \text { by setting its norm }\|u\|_{H_{0}^{\sigma}(\Omega)}=\left\|D^{\sigma} u\right\|_{L^{2}\left(\mathbb{R}^{N}\right)^{N}}, \quad 0<\sigma<1,
$$

where the $L^{2}$ norm of the distributional Riesz fractional gradient of $u$, extended by zero in $\mathbb{R}^{N} \backslash \Omega$,

$$
D^{\sigma} u(x)=(N+\sigma-1) \gamma_{N, 1-\sigma} \int_{\mathbb{R}^{N}} \frac{u(x)-u(y)}{|x-y|^{N+\sigma}} \frac{x-y}{|x-y|} d y,
$$

must be taken in the whole $R^{N}$ and not only in $\Omega$, the bounded open set where the problem is considered.

Consequently, all the integrals involving the $D^{\sigma}$ are then taken also in the whole $\mathbb{R}^{N}$ and the condition (2.1) should be read as follows: $A=A(x): \mathbb{R}^{N} \rightarrow \mathbb{R}^{N \times N}$ is a bounded and measurable matrix, not necessarily symmetric, such that, for some $a_{*}, a^{*}>0$ and for a.e. $x \in \mathbb{R}^{N}$ and all $\xi, \eta \in \mathbb{R}^{N}$,

\footnotetext{
${ }^{1}$ Rodrigues, J. F. and Santos, L., "On nonlocal variational and quasi-variational inequalities with fractional gradient". Appl. Math. Optim. 80 (2019) 835-852.
}

The original article can be found online at https://doi.org/10.1007/s00245-019-09610-0.

José Francisco Rodrigues

jfrodrigues@ciencias.ulisboa.pt

Lisa Santos

lisa@math.uminho.pt

1 CMAFcIO - Departamento de Matemática, Faculdade de Ciências, Universidade de Lisboa, 1749-016 Lisboa, Portugal

2 CMAT and Departamento de Matemática, Escola de Ciências, Universidade do Minho, Campus de Gualtar, 4710-057 Braga, Portugal 


$$
a_{*}|\xi|^{2} \leq A(x) \xi \cdot \xi \text { and } A(x) \xi \cdot \eta \leq a^{*}|\xi||\eta| .
$$

We also need to correct the definitions of

$$
\begin{aligned}
L_{v}^{\infty}\left(\mathbb{R}^{N}\right) & =\left\{v \in L^{\infty}\left(\mathbb{R}^{N}\right): v(x) \geq v>0 \text { a.e. } x \in \mathbb{R}^{N}\right\}, \\
\Upsilon_{\infty}^{\sigma}(\Omega) & =\left\{v \in H_{0}^{\sigma}(\Omega): D^{\sigma} v \in L^{\infty}\left(\mathbb{R}^{N}\right)^{N}\right\} \\
\mathbb{K}_{g}^{\sigma} & =\left\{v \in H_{0}^{\sigma}(\Omega):\left|D^{\sigma} v\right| \leq g \text { a.e. in } \mathbb{R}^{N}\right\} .
\end{aligned}
$$

and to justify the following inclusions.

Proposition For any $g \in L_{\nu}^{\infty}\left(\mathbb{R}^{N}\right)$,

$$
\mathbb{K}_{g}^{\sigma} \subset \Upsilon_{\infty}^{\sigma}(\Omega) \subset \mathscr{C}^{0, \beta}(\bar{\Omega}) \subset L^{\infty}(\Omega)
$$

for all $0<\beta<\sigma$, where $\mathscr{C}^{0, \beta}(\bar{\Omega})$ is the space of Hölder continuous functions with exponent $\beta$ and the estimate

$$
\|u\|_{L^{\infty}(\Omega)} \leq \kappa\|u\|_{H_{0}^{\sigma}(\Omega)}
$$

holds, where $\kappa>0$ depends on $\Omega$ through the Sobolev imbeddings and on $\left\|D^{\sigma} u\right\|_{L^{\infty}\left(\mathbb{R}^{N}\right)}$.

Proof For $u \in \Upsilon_{\infty}^{\sigma}(\Omega)$, as $H_{0}^{\sigma}(\Omega) \subset L^{2^{*}}(\Omega)$ and recalling that $u=0$ on $\mathbb{R}^{N} \backslash \Omega$, with the estimate

$$
\int_{R^{N}}\left|D^{\sigma} u\right|^{p} \leq\left\|D^{\sigma} u\right\|_{L^{\infty}\left(\mathbb{R}^{N}\right)^{N}}^{p-2} \int_{\mathbb{R}^{N}}\left|D^{\sigma} u\right|^{2}, \quad \forall 2<p<\infty,
$$

we obtain $u \in L^{\sigma, 2^{*}}\left(\mathbb{R}^{N}\right) \subset L^{2^{* *}}\left(\mathbb{R}^{N}\right)$, where $2^{* *}=\frac{2^{*} N}{N-2^{*} \sigma}$ if $2^{*} \sigma<N$ and $2^{* *} \geq 2$ is any finite number if $2^{*} \sigma \geq N$, by using Sobolev imbeddings. Iterating with a bootstrap argument, we obtain $u \in L^{\sigma, q}\left(\mathbb{R}^{N}\right)^{N}$, for any $q>\frac{N}{\sigma}$, and then by Morrey estimates also $u \in \mathscr{C}^{0, \beta}(\bar{\Omega})$, with $\beta=\sigma-\frac{N}{q}$.

With this estimate, all the proofs of the paper remain essentially the same with simple adaptations. For completion, we restate the main theorems on variational inequalities and the reader may check the details in the corrected pre-print available in https:// arxiv.org/pdf/1903.02646.pdf.

Theorem 2.1 Assume that $f_{i} \in L^{1}(\Omega)$ and $g_{i} \in L^{\infty}\left(\mathbb{R}^{N}\right), g_{i} \geq 0$, for $i=1,2$. Then there exists a unique solution $u_{i}$ to

$$
u_{i} \in \mathbb{K}_{g_{i}}^{\sigma}: \quad \int_{\mathbb{R}^{N}} A D^{\sigma} u_{i} \cdot D^{\sigma}\left(v-u_{i}\right) \geq \int_{\Omega} f_{i}\left(v-u_{i}\right), \quad \forall v \in \mathbb{K}_{g_{i}}^{\sigma},
$$

such that

$$
u_{i} \in \mathbb{K}_{g_{i}}^{\sigma} \cap \mathscr{C}^{0, \beta}(\bar{\Omega}), \quad \text { for all } 0<\beta<\sigma
$$


When $g_{1}=g_{2}$, the solution map $L^{1}(\Omega) \ni f \mapsto u \in H_{0}^{\sigma}(\Omega)$ is Lipschitz continuous, i.e., for some $C_{1}=\kappa / a_{*}>0$, we have

$$
\left\|u_{1}-u_{2}\right\|_{H_{0}^{\sigma}(\Omega)} \leq C_{1}\left\|f_{1}-f_{2}\right\|_{L^{1}(\Omega)} .
$$

Moreover, if in addition $f_{i} \in L^{2^{\#}}(\Omega), i=1,2$, where we set $2^{*}=\frac{2 N}{N-2 \sigma}$ and $2^{\#}=$ $\frac{2 N}{N+2 \sigma}$ when $\sigma<\frac{N}{2}$, and if $N=1$ we denote $2^{*}=q, 2^{\#}=q^{\prime}=\frac{q}{q-1}$ when $\sigma=\frac{1}{2}$ and $2^{*}=\infty, 2^{\#}=1$ when $\sigma>\frac{1}{2}$, and $g_{1}=g_{2}$, then $L^{2^{\#}}(\Omega) \ni f \mapsto u \in H_{0}^{\sigma}(\Omega)$ is Lipschitz continuous:

$$
\left\|u_{1}-u_{2}\right\|_{H_{0}^{\sigma}(\Omega)} \leq C_{\#}\left\|f_{1}-f_{2}\right\|_{L^{2^{\#}(\Omega)}},
$$

for $C_{\#}=C_{*} / a_{*}>0$, where $C_{*}$ is the constant of the Sobolev embedding $H_{0}^{\sigma}(\Omega) \hookrightarrow$ $L^{2^{*}}(\Omega)$.

Theorem 3.1 If $g \in L_{v}^{\infty}\left(\mathbb{R}^{N}\right)$ and $f \in L^{2^{\#}}(\Omega)$, then the Lagrange multipliers problem

$$
\begin{gathered}
\left\langle\lambda D^{\sigma} u, D^{\sigma} v\right\rangle_{\left(L^{\infty}\left(\mathbb{R}^{N}\right)^{N}\right)^{\prime} \times L^{\infty}\left(\mathbb{R}^{N}\right)^{N}}+\int_{\mathbb{R}^{N}} A D^{\sigma} u \cdot D^{\sigma} v=\int_{\Omega} f v, \quad \forall v \in \Upsilon_{\infty}^{\sigma}(\Omega), \\
\left|D^{\sigma} u\right| \leq g \text { a.e. in } \mathbb{R}^{N}, \lambda \geq 0 \text { and } \lambda\left(\left|D^{\sigma} u\right|-g\right)=0 \text { in } L^{\infty}\left(\mathbb{R}^{N}\right)^{\prime}
\end{gathered}
$$

has a solution

$$
(\lambda, u) \in L^{\infty}\left(\mathbb{R}^{N}\right)^{\prime} \times \Upsilon_{\infty}^{\sigma}(\Omega)
$$

Moreover, $u$ solves the variational inequality of Theorem 2.1.

In the last section on quasi-variational inequalities, where the nonlinear map for the threshold $g=G[u]$, depending on the solution $u$, has now image in $L_{v}^{\infty}\left(\mathbb{R}^{N}\right)$, the results are also almost unchanged, with the exception of the Theorem 4.3, which is improved by the estimate of the above proposition, essentially with the same proof (see https://arxiv.org/pdf/1903.02646.pdf). It reads now in the following form.

Theorem 4.3 Let $f \in L^{1}(\Omega)$, as in Theorem 2.1, and the functional $G$ be such that

$$
G: \mathscr{C}^{0}(\bar{\Omega}) \rightarrow L_{v}^{\infty}\left(\mathbb{R}^{N}\right) \quad \text { is a continuous operator. }
$$

Then there exists a solution of the quasi-variational inequality

$$
\begin{aligned}
& u \in \mathbb{K}_{G[u]}^{\sigma}=\left\{v \in H_{0}^{\sigma}(\Omega):\left|D^{\sigma} v\right| \leq G[u] \text { a.e. in } \mathbb{R}^{N}\right\} \\
& \int_{\mathbb{R}^{N}} A D^{\sigma} u \cdot D^{\sigma}(v-u) \geq \int_{\Omega} f(v-u), \quad \forall v \in \mathbb{K}_{G[u]}^{\sigma} .
\end{aligned}
$$

Publisher's Note Springer Nature remains neutral with regard to jurisdictional claims in published maps and institutional affiliations. 